TITLE:

\title{
Applications of time-domain spectroscopy to electron-phonon coupling dynamics at surfaces.
}

\author{
$\operatorname{AUTHOR}(S)$ :
}

Matsumoto, Yoshiyasu

\section{CITATION:}

Matsumoto, Yoshiyasu. Applications of time-domain spectroscopy to electron-phonon coupling dynamics at surfaces.. Chemical record 2014, 14(5): 952-963

\section{ISSUE DATE:}

2014-10

URL:

http://hdl.handle.net/2433/202092

\section{RIGHT:}

This is the peer reviewed version of the following article: Matsumoto, Y. (2014), Applications of Time-Domain

Spectroscopy to Electron-Phonon Coupling Dynamics at Surfaces. Chem. Rec., 14: 952-963, which has been published in final form at http://dx.doi.org/10.1002/tcr.201402030. This article may be used for non-commercial purposes in accordance with Wiley Terms and Conditions for Self-Archiving.: The full-text file will be made open to the public on 19 AUG 2015 in accordance with publisher's 'Terms and Conditions for Self-Archiving'. This is not the published version. Please cite only the published version.; この論文は出版社版でありません。引用の際には出版社版をご確認ご利用くだ 


\title{
Applications of Time-Domain Spectroscopy to Electron-Phonon Coupling Dynamics at Surfaces
}

\author{
YOSHIYASU MATSUMOTO \\ Kyoto University, Graduate School of Science, Department of Chemistry, Kyoto 606-8502 \\ (Japan) \\ E-mail: matsumoto@kuchem.kyoto-u.ac.jp
}

\begin{abstract}
:
Photochemistry is one of the most important branches in chemistry to promote and control chemical reactions. In particular, there has been growing interest in photoinduced processes at solid surfaces and interfaces with liquid such as water for developing efficient solar energy conversion. For example, photoinduced charge transfer between adsorbates and semiconductor substrates at the surfaces of metal oxides induced by photo-generated holes and electrons is a core process in photovoltaics and photocatalysis. In these photoinduced processes, electron-phonon coupling plays a central role. This paper describes how time-domain spectroscopy is applied to elucidate electron-phonon coupling dynamics at metal and semiconductor surfaces. Because nuclear dynamics induced by electronic excitation through electron-phonon coupling take place in the femtosecond time domain, the pump-and-probe method with ultrashort pulses used in the time-domain spectroscopy is a natural choice for elucidating the electron-phonon coupling at metal and semiconductor surfaces. Starting with a phenomenological theory of coherent phonon generated by impulsive electronic excitation, this paper describes a couple of illustrative examples of the applications of linear and nonlinear time-domain spectroscopy to a simple adsorption system, alkali metal on $\mathrm{Cu}(111)$, and more complex systems of photocatalysts.
\end{abstract}

Keywords: electron-phonon coupling, coherent phonon, photocatalysis, alkali atom, titanium dioxide, bismuth vanadate, second harmonic generation, transient absorption 


\section{Introduction}

Electron-phonon coupling is not only the central issue in the electrical and thermal properties of solids including superconductivity, but also plays an crucial role in energy dissipation, charge transfer, and chemical reactions at surfaces and interfaces. In particular, nonthermal electronic excitation by irradiation of photons and electrons induces motions of adsorbate nuclei and ions in the lattice of substrate, which are the primary steps of photochemistry and photocatalytic reactions. Therefore, it is of paramount importance to understand the nature of electron-phonon interaction and how the interaction manifests itself in various properties and processes at surfaces and interfaces.

A schematic representation depicted in Fig. 1 shows how the nonadiabatic coupling is involved in adsorbate nuclear motions at metal surfaces. When an adsorbate comes closer to the surface, the unoccupied state of adsorbate is shifted and broadened by the interaction with substrate electronic states, and ultimately the electronic state is partially filled at the equilibrium position on the surface. When the adsorbate oscillates around the equilibrium position, this partially filled band moves up and down in energy, responding to the adsorbate nuclear motion; this modulates the degree of occupation in the adsorbateinduced state at the oscillation frequency. In this way, the nuclear motions and charge transfer at the surface is closely coupled. Moreover, intra-adsorbate vibrations also couple to electrons in the continuum band of metal; this provides another route for vibrational relaxation in addition to phonon-phonon coupling. ${ }^{[1,2]}$

With the order reversed, nonthermal excitation of substrate electrons can excite intraadsorbate vibrational modes and modes between adsorbate and substrate. When the substrate is electronically excited with a short laser pulse, the population of energetic electrons, i.e., hot electrons, increases. Scattering and temporal occupation of hot electron in the partially filled adsorbate-induced state bring the adsorbate in a new electronic state, i.e, a transient anionic state; thus, adsorbate nuclei move to adapt new equilibrium positions, resulting in vibrational excitation. This nonthermal vibrational excitation can be understood as an outcome of electronic heating of adsorbate vibrational modes and it has been well treated by the electron friction model. ${ }^{[3-8]}$ 


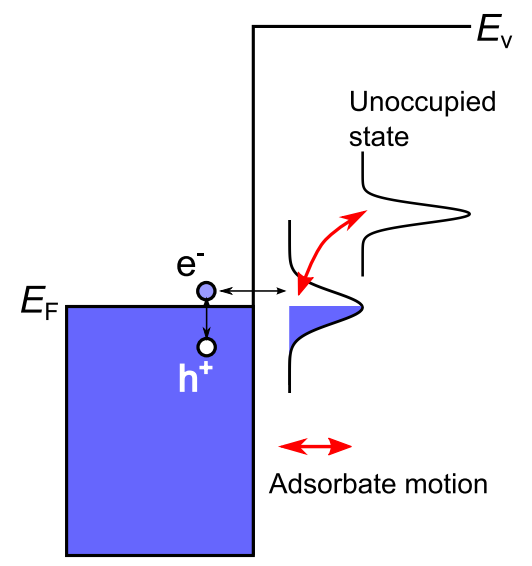

Figure 1: Illustrative representation of electron-phonon coupling at a metal surface. The unoccupied state of adsorbate is shifted and broadened by the interaction with metal as the adsorbate approaches to the surface. The density of electron occupied in the adsorbateinduced band depends on the displacement of adsorbate from the metal surface. $E_{F}$ and $E_{V}$ denote the Fermi and vacuum levels, respectively.

In the case of semiconductors, such as metal oxides that are useful materials for photovoltaics and photocatalysis, electronic excitation across the band gap of metal oxide creates electrons in the conduction band and holes in the valence band. Because the charge density distribution around ions in the lattice is modified by the electronic excitation, the lattice of metal oxide is deformed in the electronic excited state, so that phonon modes are excited through electron-phonon coupling. Photo-generated charges relax rapidly to each of the band edges and some fractions of electrons and holes are localized at trap sites. When charges are trapped at the surface of metal oxide, the trapped charges not only deform the lattice around them, but also affect the distance to and orientation of molecules at the interface. These changes in molecular configurations with respect to the surface of metal oxide are one of the key factors controlling charge transfer rates at the interface. Therefore, the electron-phonon couplings in metal oxide play an important role in charge dynamics and charge transfer at the interface.

The effects of electron-phonon couplings at surfaces and interfaces on energy and charge transfer can be studied with frequency-domain spectroscopy. For example, the lineshape 
analysis in infrared (IR) absorption spectroscopy has provided the information of electronphonon coupling in vibrational relaxation of adsorbate. ${ }^{[1,4,9-11]}$ However, because electronic excitation and subsequent charge dynamics are inherently ultrafast processes, it is natural and advantageous to employ time-domain spectroscopy to explore the lattice and adsorbate motions through electron-phonon coupling. ${ }^{[12-14]}$

This paper describes a perspective of the author on photo-induced electron-phonon couplings mainly focusing on surfaces of metal and metal oxide. Optical responses right after electronic excitation with an ultrashort pump pulse provide us the information how nuclear motions are induced by the electronic excitation. Section 2 provides a framework of coherent phonon excitation induced by electronic excitation with ultrashort laser pulses. Section 3 describes an illustrative example of coherent phonon of alkali metal adsorbates on metal surfaces. In Section 4, a couple of examples are given on coherent surface lattice phonons of photocatalysts induced by irradiation of ultrashort laser pulses. Finally, concluding remarks and outlook are given in Section 5.

\section{Framework for coherent phonon excitation}

First, let us introduce a phenomenological description of coherent phonon in terms of classical mechanics. If a force $F(t)$ exerted to the lattice composed of oscillators with the effective mass $\mu$ has a duration or a rapid rising edge shorter than the oscillation period of a phonon mode, a phonon mode is excited coherently i.e., in phase. The equation of motion for the displacement of coherent oscillation can be described as:

$$
\mu\left[\frac{d^{2} Q}{d t^{2}}+2 \beta \frac{d Q}{d t}+\Omega_{0}^{2} Q\right]=F(t),
$$

where $\Omega_{0} / 2 \pi$ is the frequency of an undamped oscillator and $\beta$ is the damping rate of the oscillator. Here we assume that the oscillators interact with a bath system. Thus, the coherent phonon is damped by population decay and pure dephasing as a result of interactions with the bath system. The formal solution of eq. (1) can be written as:

$$
Q(t)=\frac{1}{\mu \Omega_{1}} \int_{-\infty}^{t} d t^{\prime} F\left(t^{\prime}\right) e^{-\beta\left(t-t^{\prime}\right)} \sin \left[\Omega_{1}\left(t-t^{\prime}\right)\right]
$$


where $\Omega_{1}=\sqrt{\Omega_{0}^{2}-\beta^{2}}$.

When the force is turned off, the system shows a free-induction decay with a damping rate $\beta$ :

$$
Q(t) \propto \cos \left(\Omega_{1} t-\phi\right) e^{-\beta t}
$$

where $\phi$ is the initial phase. In general, the initial phase is described as: ${ }^{[15]}$

$$
\tan \phi=\frac{\operatorname{Im}\left[i \tilde{F}\left(-\Omega_{1}-i \beta\right)\right]}{\operatorname{Re}\left[i \tilde{F}\left(-\Omega_{1}-i \beta\right)\right]} .
$$

Here, $\tilde{F}(\Omega)$ is the Fourier transform of $F(t)$. Thus, we obtain the relationship between the initial phase and the temporal profile of the force: if the force is impulsive, i.e., $F(t) \propto \delta(t)$, the initial phase is $\pi / 2$ : the nuclear oscillation is described as $Q(t) \propto \exp (-\beta t) \sin \Omega_{1} t$; if the force has a finite duration, the initial phase deviates from $\pi / 2$, depending on the temporal profile of $F(t)$. Therefore, the initial phase provides useful information of the time profile of $F(t)$.

As shown in Fig. 2, there are a couple of excitation mechanisms of coherent phonon. First, when the bandwidth of a pump laser pulse is larger than a phonon frequency, photons in the pump pulse can excite the phonon mode via virtual electronic excitation: impulsive stimulated Raman scattering (ISRS) (Fig. 2(a)). According to eq. (4), the initial phase of coherent phonons in this case is $\pi / 2$; the coherent motion is described as a sine function. Second, when the photon energy of a pump pulse becomes near resonant to an electronic transition, ISRS excitation is strongly enhanced (Fig. 2(b)). The initial phase depends on the detuning of the photon energy with respect to an electronic transition of the system and the electronic dephasing time. ${ }^{[16]}$ Third, when the photon energy of a pump laser pulse is resonant to an electronic transition, the ultrafast-laser pulse creates a timedependent excited-carrier distribution (Fig. 2(c)). The transient changes in the electron density distribution displace ions in the lattice of solid. If the leading edge of changes in the electron density distribution contains Fourier components whose frequencies exceed the phonon frequency, the phonon mode is excited in phase. This mechanism is denoted as displacive excitation of coherent phonons (DECP). ${ }^{[17]}$ In contrast to the stimulated Raman scattering, the initial phase is not $\pi / 2$ because of a finite duration of the force. 


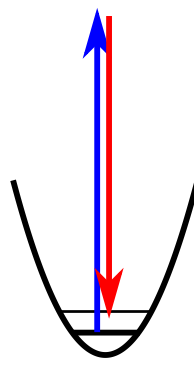

(a)



(b) (c)

Figure 2: Excitation mechanisms for coherent phonon: (a) nonresonant impulsive stimulated Raman excitation, (b) resonant resonant impulsive stimulated Raman excitation, and (c) resonant displacive excitation.

In the case of real excitation of electrons in solid, the initial phase of coherent phonon depends on the time profile of the distribution of electrons that affects the bonding in the lattice. If the electron distribution persists much longer than the period of a phonon mode, the force exerted on ions has a time profile like a step function; in this case the initial phase of coherent phonon is close to 0 or $\pi$, so that coherent phonon decays as a cosine damped oscillator. If the electron distribution decays much faster than the period of a phonon mode, lattice ion motions are impulsively activated, so that the coherent phonon decays as a sine damped oscillator. Riffe et al. ${ }^{[15]}$ have established a unified treatment of initial phase of coherent phonon in terms of the temporal profile of force exerted on ions of the lattice.

The idea of coherent phonon initiated by rapid electronic excitation can be applied to the phonon modes near the surface of solid and adsorbates on the surface. Ultrafast screening of the surface depletion field by photoexcited carriers creates LO-phonon-plasmon modes. ${ }^{[18]}$ Coherently excited phonon modes were observed on various semiconductor surfaces including GaAs, ${ }^{[19,20]} \mathrm{InN},{ }^{[21]}$ and $\mathrm{Bi}_{2} \mathrm{Te}_{3} .{ }^{[22]}$ As described in Introduction, at metal surfaces, the empty electronic level of molecule hybridized with metal electronic states as the molecule approaches the surface. Upon irradiation of a short laser pulse, electron-hole pairs are created at and near the surface by absorption of incident photons. 
Excited electrons are resonantly scattered at the adsorbate to fill the partially-filled band. This causes attractive or repulsive force between adsorbate and surface, depending of the bonding nature of the partially filled state. If a duration of force is shorter than the oscillating period of a vibrational mode of adsorbate, the transient electronic excitation also initiates coherent surface phonons. Because absorption cross sections are usually much larger than Raman scattering cross sections on metal surfaces, real electronic excitations with ultrashort laser pulses play a dominant role in creating coherent phonons at metal surfaces; hence, it is important to specify which electronic transition, between bulk bands or adsorbate-induced bands, really induces adsorbate nuclear motions. One of the important issues in the study of coherent surface phonons at metal surfaces is to clarify the origin of the force responsible for the coherent excitation of phonons.

\section{Coherent phonons of alkali-metal layers at metal surfaces}

Alkali-metal overlayers on metal surfaces are one of the prototypes of chemisorption systems. Thus, the geometric and electronic structures of alkali-covered metal surfaces have been one of the central issues in surface science. ${ }^{[23]}$ Most of alkali atoms form hexagonal structures on metal surfaces of fcc(111) and even on surfaces with square or rectangular symmetry of fcc(001). At low coverages, alkali atoms donate electrons to metal substrate; thus, the adsorbates are cationic and the work function of the metal substrate is substantially reduced. At high coverages, the $\mathrm{Cu}$ overlayers are metallic, because the orbitals of neighboring alkali atoms overlap extensively with each other; thus, the work function of the adsorption system becomes close to that of alkali metal. What is fascinating in the alkali adsorption system is that we can control the electronic structure by changing alkali coverage.

Here, we focus on a specific system: $\mathrm{Cs}$ on $\mathrm{Cu}(111)$. Cs atoms adsorb on $\mathrm{Cu}(111)$ to form overlayers without massive reconstruction. One of the most interesting features in the electronic structure of $\mathrm{Cs}$ overlayer on $\mathrm{Cu}(111)$ is that the $\mathrm{Cs}$ overlayer forms a two-dimensional quantum well. This is because a $\mathrm{Cu}(111)$ surface has an $s, p$-inverted $L$ projected band gap. Thus, a two-dimensional quantum well is formed between the barrier 




Figure 3: Electronic structure of an alkali-covered $\mathrm{Cu}(111)$ surface at the full coverage. QWS: quantum-well state, and OR: overlayer resonance. The shaded regions show the band structure of bulk copper projected onto the surface Brillouin zone.

at the $\mathrm{Cu}(111)$ surface and the barrier toward vacuum. As shown in Fig. 3, two bands characteristic of the Cs overlayer are an overlayer resonance (OR) located below the $L$-band gap and a quantum well state (QWS) at around $E_{\mathrm{F}}$ in the band gap around the $\bar{\Gamma}$ point. These bands correlate to those of a free-standing alkali monolayer in the vacuum: the $s$-like lowest and the $p_{z}$-like second lowest bands. ${ }^{[24]}$ When the monolayer is brought closer to the metal surface, they are stabilized by the interaction with the metal, while these bands maintain the integrity. The $s$-like band correlates to OR; the $p_{z}$-like band correlates to QWS. Because QWS is located in the $L$-band gap, its wave function is localized at the surface. In contrast, the wave function of OR extends deeper into the substrate, because it is located below the lower edge of $L$-band gap.

When a metal surface covered with alkali metal atoms is electronically excited by ultrashort laser pulses, the stretching motions of alkali atoms with respect to the metal surface are excited coherently. Our group have explored the nuclear dynamics of various alkali metal atoms adsorbed on metal surfaces, ${ }^{[25-33]}$ using time-resolved second harmonic gen- 
eration (TRSHG). Second harmonic generation (SHG) is a second-order nonlinear optical process and has been used as a surface sensitive spectroscopy, because SHG signals are generated only at the surface where the centrosymmetry of bulk crystal is broken. ${ }^{[34]}$

TRSHG is particularly suitable for studies of alkali adsorbates, because SHG signals are dramatically enhanced with alkali metal adsorption due to multipole plasmon excitation at high coverage. ${ }^{[35]}$ In TRSHG spectroscopy, the SH intensity of a probe pulse is measured as a function of pump-probe delay time $t$. Transient changes in the SH intensity $\Delta I_{\mathrm{SH}}(t)$ are defined as $\Delta I_{\mathrm{SH}}(t)=\left[I_{\mathrm{SH}}(t)-I_{\mathrm{SH}}^{0}(t)\right] / I_{\mathrm{SH}}^{0}(t)$, where $I_{\mathrm{SH}}(t)$ and $I_{\mathrm{SH}}^{0}(t)$ are the $\mathrm{SH}$ intensities at a delay time $t$ with and without a pump pulse, respectively. From now on, we denote $\Delta I_{\mathrm{SH}}(t)$ as TRSHG signals.

Figure 4(a) shows typical traces of TRSHG signals. Both excitations at the wavelengths $\lambda_{\mathrm{ex}}=400$ and $800 \mathrm{~nm}$ generate clear oscillatory modulations in the TRSHG signals. These modulations originate from coherent surface phonons of Cs-Cu stretching; the stretching motion modulates the second-order nonlinear susceptibility, $\chi^{(2)}$, through electron-phonon coupling at the surface. The Fourier-transformed power spectra in the inset of Figure 4(a) show that the frequency of Cs-Cu stretching is $\nu=1.8 \mathrm{THz}$. Although there is little difference in frequency between excitations at $\lambda_{\mathrm{ex}}=400$ and $800 \mathrm{~nm}$, the initial phase of the oscillating component at $\lambda_{\mathrm{ex}}=800 \mathrm{~nm}$ is very different from that at $\lambda_{\mathrm{ex}}=400 \mathrm{~nm}$, as shown in Fig. 4(b). As described in Section 2, the initial phase provides us the information of the temporal profile of force driving the coherent vibration. Fitting of the TRSHG traces to eq. (3) determined the initial phases: $\phi=(-84 \pm 1)^{\circ}$ at $\lambda_{\mathrm{ex}}=800 \mathrm{~nm}$ and $(-149 \pm 1)^{\circ}$ at $\lambda_{\mathrm{ex}}=400 \mathrm{~nm}$. Thus, Cs-Cu stretching is sin-like at $\lambda_{\mathrm{ex}}=800 \mathrm{~nm}$, while it is close to cos-like at $\lambda_{\text {ex }}=400 \mathrm{~nm}$.

Let us discuss what electronic transitions are responsible for the generation of coherent Cs-Cu stretching oscillations showing the distinct excitation wavelength dependence of initial phase. The electron density around a Cs adatom has to be modulated to generate the force field driving its coherent stretching motion. The electronic transitions involved in adsorbate-induced electronic states are effective in doing so. The unoccupied Cs $5 d$ band was reported to be at $\sim 1.6 \mathrm{eV}$ in inverse photoemission measurements. ${ }^{[36]}$ Furthermore, 




Figure 4: (a) Excitation wavelength dependence of TRSHG traces taken from $\mathrm{Cs} / \mathrm{Cu}(111)$ at $\lambda_{\mathrm{ex}}=800 \mathrm{~nm}$ (red open circles) and $\lambda_{\mathrm{ex}}=400 \mathrm{~nm}$ (blue open squares). The probe wavelength is $565 \mathrm{~nm}$ for both traces. The incident pump fluence was $8.0 \mathrm{~mJ} / \mathrm{cm}^{2}$ and the Cs coverage was $0.23 \mathrm{ML}$ at $\lambda_{\mathrm{ex}}=800 \mathrm{~nm} ; 3.6 \mathrm{~mJ} / \mathrm{cm}^{2}$ and $0.25 \mathrm{ML}$ at $\lambda_{\mathrm{ex}}=400 \mathrm{~nm}$. Solid lines are results of the nonlinear least-square fitting with eq. (3). Dotted lines are overdamped components contributed by hot electrons. (Inset) Fourier power spectra of the oscillatory components of the TRSHG traces for $\lambda_{\mathrm{ex}}=800 \mathrm{~nm}$ (solid) and for $\lambda_{\mathrm{ex}}=400 \mathrm{~nm}$ (dotted). (b) Oscillating components obtained by subtracting the overdamped ones. Note that initial phases are very different to each other, while the frequencies are very similar. Reprinted with permission from Ref. ${ }^{[33]}$ Copyright 2011 American Chemical Society.

the third image potential state (IPS) at $\bar{\Gamma}$ is also located at $\sim 1.6 \mathrm{eV}$ according to density functional theory calculations. ${ }^{[37]}$ Because QWS is located near $E_{\mathrm{F}}$, the transitions from QWS to these unoccupied surface localized states are near resonant to the photon energy at $\lambda_{\mathrm{ex}}=800 \mathrm{~nm}: \mathrm{h} \nu=1.55 \mathrm{eV}$. Although 800-nm photons are also absorbed by the bulk transitions in the $s, p$-band, the oscillator strength of the bulk transitions could be substantially smaller than the transition between adsorbate-localized states, because the density of states of $s, p$-band involved in the bulk transitions at $800 \mathrm{~nm}$ is small. Thus, we assigned the near resonant transition from QWS to either the Cs $5 d$ band or the third IPS to be responsible for the generation of coherent $\mathrm{Cs}-\mathrm{Cu}$ stretching. In this case, $\mathrm{Cs}$ is 
excited locally and quenched very rapidly because of effective resonant charge transfer between the adsorbate-localized bands and substrate bands. Thus, the excited lifetime could be very short. Consequently, the force exerted to Cs is regarded as impulsive compared with a period of $\mathrm{Cs}-\mathrm{Cu}$ stretching, resulting in the sin-like oscillation.

In contrast, substrate excitation is more substantial at $\lambda_{\mathrm{ex}}=400 \mathrm{~nm}$ than at $\lambda_{\mathrm{ex}}=800$ $\mathrm{nm}$, because 400-nm photons can excite electrons in the filled $d$-bands of $\mathrm{Cu}$ in addition to those in the $s, p$-band. Upon excitation by fs pump pulses with a fluence of $20 \mathrm{~mJ} / \mathrm{cm}^{2}$ at $\lambda_{\mathrm{ex}}=400 \mathrm{~nm}$, the substrate electron temperature increases to several thousand $\mathrm{K}$ within 0.1 ps and decays to $1000 \mathrm{~K}$ in $\sim 1$ ps according to calculations based on the two-temperature model. ${ }^{[38]}$ Electron transfer from the substrate to the adsorbate-localized states takes place, while a bath of substrate electrons maintains its temperature high enough for electrons to transfer to the adsorbate unoccupied states. This implies that the electron temperature increases with a very sharp rising edge and stays high in a much longer time interval than a period of $\mathrm{Cs}-\mathrm{Cu}$ stretching. Thus, the force exerted to Cs has a temporal profile close to a step function. Consequently, the substrate excitation is believed to be responsible for generation of coherent phonons with the cos-like oscillation.

This illustrative example, $\mathrm{Cs}$ atoms on $\mathrm{Cu}(111)$, shows that the alkali-induced electronic state i.e., QWS, plays an central role in generating photoinduced coherent nuclear motions of adsorbates. This adsorption system is a rare case where the detailed discussion on how adsorbate nuclear motions are induced with a specific electronic excitation, since the electronic structure of alkali covered metal surface is relatively well known over a wide range of coverage.

The time-domain method not only provides information of coherent phonons, but also allows us to control phonon modes to be excited. Here we show how to excite a phonon mode selectively with a burst of pump pulse trains. ${ }^{[27]}$ There are two phonon modes excited by ultrashort laser pulses in the case of Cs adsorbed on Pt(111): Cs-Pt stretching and the Rayleigh phonon modes of Pt substrate excited. The frequencies of the two components are very close: $2.3 \mathrm{THz}$ for the Cs-Pt stretching and $2.7 \mathrm{THz}$ for the Pt substrate mode. ${ }^{[25,26,28]}$

We prepared pulse trains for pumping electronically the $\mathrm{CO} / \mathrm{Pt}(111)$ adsorption system 
(a)

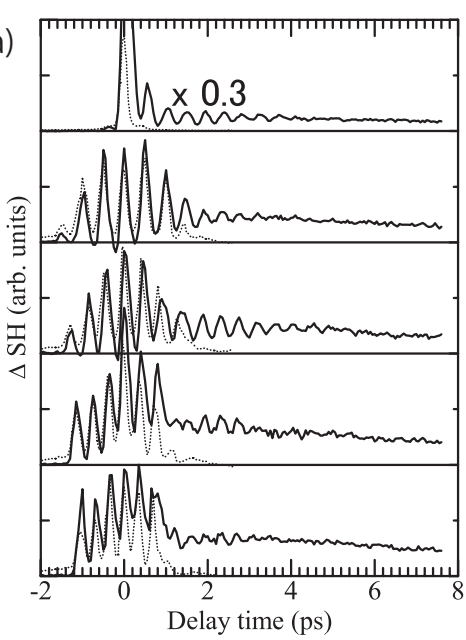

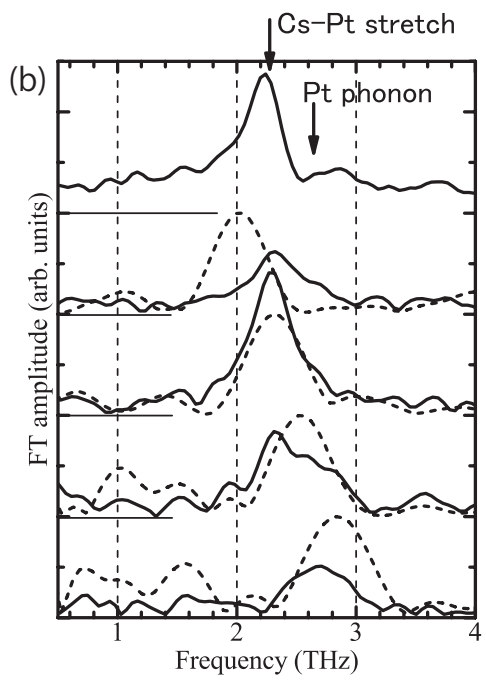

Figure 5: (a) TRSHG traces and (b) Fourier transformed spectra of Cs deposited on $\mathrm{Pt}(111)$. The result at the top panel was obtained by single pulse excitation. The results from the second top to the bottom panel were obtained by varying the repetition rate from 2.0, 2.3, 2.6, and $2.9 \mathrm{THz}$. The gray lines in (a) are the time profiles of excitation pulse trains. The dashed curves in (b) are the Fourier spectra of the cross-correlation traces of the excitation pulse trains. Adapted from Ref. ${ }^{[27]}$ with permission from the PCCP Owner Societies.

by employing the pulse shaper consisting of a liquid-crystal spatial light modulator, a pair of gratings, and cylindrical lenses in a $4 \mathrm{f}$ configuration, where the optical components are placed at a distance of one focal length $(f)$ to each other. ${ }^{[39]}$ With this optical setup, we tuned the repetition rate of pulse trains. Fig. 5 shows a series of TRSHG traces and their Fourier-transformed spectra, respectively, by pulse train excitation as a function of the repetition rate in the range from 2.0 to $2.9 \mathrm{THz}$. Note that the Fourier spectra of the TRSHG traces depend on the repetition rate of pulse trains. At $2.3 \mathrm{THz}$, a peak at the same frequency is strongly enhanced and the dip due to the Pt surface phonon mode is absent. As the repetition rate is increased, the peak intensity at $2.3 \mathrm{THz}$ decreases while the one at the higher frequency component increases. When the repetition rate is tuned to $2.9 \mathrm{THz}$, the $2.3 \mathrm{THz}$ component is almost completely quenched and only a peak at 
2.7 THz appears, which corresponds to the Pt phonon mode. In this way, it is possible to selectively excite either the Cs-Pt stretching mode or the Rayleigh phonon mode by tuning the repetition rate.

This work is a demonstration of controlling excitation of phonon modes by the irradiation of pulse trains, but this method does not use optical phase to control selective excitation. Very recently, we have reported that the degrees of excitation between two different bulk phonon modes of Bi can be controlled by using optical phase of pump pulses. ${ }^{[40]}$

\section{Coherent phonons of metal oxides: photocatalysis}

Heterogeneous photocatalysis has attracted much attention because of its applications to solar energy conversion. In particular, much effort has been devoted to photocatalytic water splitting since the discovery of photoelectrochemical water decomposition with $\mathrm{TiO}_{2},{ }^{[41]}$ because it provides a means of artificial photosynthesis without any burden to the environment. ${ }^{[42-45]}$ Heterogeneous photocatalysts need to have multiple essential capabilities: harvesting light, transporting charges, and catalyzing reactions. Thus, as shown in Fig. 6, heterogeneous photocatalysis with semiconductor-based powders is composed of a couple of major processes: generation of electron-hole pairs by optical transitions across the band gap of semiconductor, charge separation and transportation in semiconductor, and redox reactions at semiconductor surfaces.

Among these processes, we focus here on the creation of electron-hole pairs and subsequent charge and lattice dynamics. Upon excitation across the band gap of a metal oxides, the potential energy surface of ions in the lattice change. Thus, the ions in the lattice are displaced from the equilibrium points in the ground state. When electron-hole pairs are created with ultrafast laser pulses, the impulsive force initiated by the electronic excitation generate coherent phonons in the metal oxides.

An example of the optical coherent phonon creation has been recently demonstrated by Bothschafter et al. ${ }^{[46]}$ They have observed the generation of a coherent optical phonon of $\mathrm{TiO}_{2}$ upon the resonant excitation with ultrashort laser pulses at $245 \mathrm{~nm}$. As shown in Fig. $7(\mathrm{~b})$, the signals of $\mathrm{A}_{1 \mathrm{~g}}$ coherent phonon show a phase shift of $-0.16 \pi$ irrespective 
(3)



Figure 6: Schematic representation of basic steps of photocatalysis with semiconductorbased powders: (1) generation of electron-hole pairs by optical transitions across the band gap of semiconductor, (2) charge separation and transportation in semiconductor, and (3) redox reactions at semiconductor surfaces.

to pump fluence. This phase shift corresponds to a temporal delay of $\sim 5.5 \mathrm{fs}$. With the aid of calculations of the excited-state potential energy surface (PES) and the motion of lattice ions on the time-dependent PES, they interpreted that the phase shift is caused by the time-evolution of PES after the resonant excitation. The resonant excitation from the valence band to the conduction band of $\mathrm{TiO}_{2}$ induces electron transfer from $\mathrm{O}^{2-}$ to $\mathrm{Ti}^{3+}$, because the valence and conduction bands have the characters of $\mathrm{O} 2 \mathrm{p}$ and $\mathrm{Ti} 3 \mathrm{~d}$, respectively. Thus, the optical excitation induces a unidirectional shift of the PES along the $A_{1 g}$ optical phonon coordinate (Fig. 7(a)). If this shift of the PES were solely responsible for the generation of coherent phonon, i.e., through purely DECP mechanism, the initial phase would be 0 or $\pi$, i.e., purely cosine oscillation. The phase shift observed indicates that a second displacive driving mechanism operates in this system. Combining the electron dynamics with the PES calculations, they proposed that the rapid cooling of carriers also contribute to the generation of coherent phonon. Because excited electrons relax to the bottom of the conduction band much faster than the oscillation period of the $\mathrm{A}_{1 \mathrm{~g}}$ phonon, the fast dynamic shift of the PES minimum drives the phonon mode coherently.

Usually continuous light sources are used for photocatalysis, because the ultimate goal of photocatalysis is to use sun light for water splitting and degradation of organic pollutants. Electronic transitions by the continuous light source also excite phonon modes 


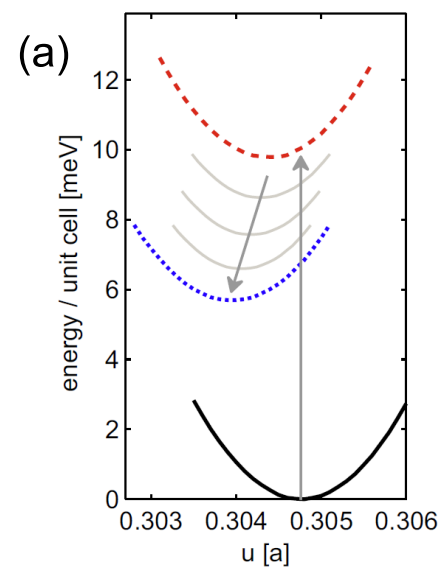

(b)

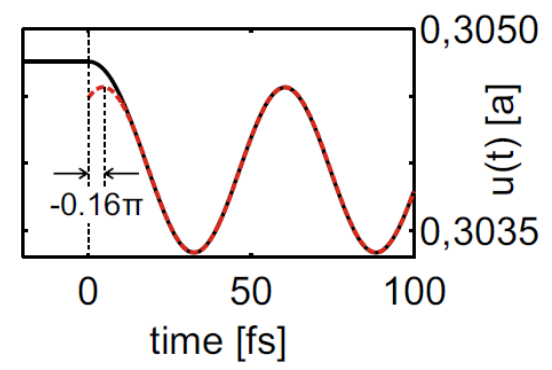

Figure 7: (a) Potential energy surface along the $A_{1 g}$ phonon coordinate in the electronic ground state (solid black line), the hot laser-excited state with an electron temperature of $1 \mathrm{eV}$ (red dashed line), and cooled excited state with a temperature of $0.027 \mathrm{eV}$. The PES of hot and cold excited states are offset by -419.9 and $-197.3 \mathrm{meV}$, respectively, for clarity of representation. (b) Calculated oxygen atom trajectory (black solid line), in comparison with a cosine oscillation at $17.9 \mathrm{THz}$ that perfectly describes the motion after $t=20 \mathrm{fs}$. Reprinted with permission from Ref. ${ }^{[46]}$ Copyright 2013 American Physical Society.

through vibronic coupling and the excitation of surface phonons of a photocatalyst may influence charge transfer between the photocatalyst and adsorbed molecules. With the continuous light, however, coherent motions of the lattice of photocatalyst are not created, because phonons are excited incoherently. Thus, coherent phonon generation may not be relevant to photocatalysis. Nonetheless, application of the time-domain spectroscopy to photocatalysts can provide useful information of electron-phonon coupling at trapped charges that play an important role in photocatalysis.

Photo-generated charges, photoelectrons and photoholes, are rapidly relaxed to the bottoms of conduction and valence bands, respectively, after the electronic excitation across the band gap of semiconductor, and they are stabilized as polarons. Some charges are localized by being trapped at the states located in the band gap of photocatalyst. Trapping of charges plays important roles in photocatalytic reactions. First, trapping of charge retards 
recombination of electron and hole. This is essential to improve the photo-conversion yield of photocatalysis, because electron-hole recombination is a major loss mechanism. Second, trapped charges are believed to be responsible for redox reactions. In particular, oxygen evolution by oxidation of water, four-electron and four-proton process, proceeds very slowly. This requires a long lifetime of holes. ${ }^{[47]}$ It has been reported that the photohole lifetime for $\mathrm{O}_{2}$ evolution on $\mathrm{TiO}_{2}$ photoanodes is estimated to be $\sim 30 \mathrm{~ms}$ at $\mathrm{pH} 12.7^{[48]}$ and $\sim 0.2 \mathrm{~s}$ at $\mathrm{pH} 6.5 .{ }^{[49]}$ Therefore, the characterization of trapping sites, such as their spatial locations, geometric structures, energy levels, the time scales of trapping, etc is vital for an understanding of the photocatalytic reaction mechanism.

Charge trapped states have been explored mainly on $\mathrm{TiO}_{2}$. Charge trap sites are attributed to oxygen vacancies, surface hydroxyl, interstitial $\mathrm{Ti}^{3+}$, etc. Trapped charges at these trap sites have been identified with various experimental methods, ${ }^{[50]}$ including uv-vis absorption, infrared, Raman, and EPR spectroscopies. Although these methods probing the steady states of photocatalysts are useful for characterizing deep trap sites, but they are not capable to probe trapping processes right after photoexcitation. Thus, time-resolved spectroscopy is necessary for probing the dynamics of charge splitting and trapping in shallow traps.

Ultrafast time-resolved spectroscopies have indicated that photo-generated charges are trapped in shallow traps in the ps time domain or shorter. ${ }^{[50]}$ Photoinduced charge dynamics has been probed by transient absorption in the wide wavelength range (from ultraviolet/visible to far infrared) and in the long time domain (from subpicosecond to second). ${ }^{[51-56]}$ Comparison of transient spectra with and without hole or electron scavengers allow to assign wavelength ranges where electrons or holes mainly contribute to transient absorption. In spite of the numerous transient spectroscopic studies, it is not clear how electronic excitation and subsequent charge trapping affect the lattice structure of photocatalyst. The deformation of lattice caused by electron-phonon coupling is responsible for the energy stabilization of charge. Thus, the electron-phonon couplings in photocatalytic systems play an important role in charge dynamics and charge transfer between photocatalyst and adsorbate. 
Nomoto et al. ${ }^{[57,58]}$ have observed coherent phonon generation in bulk $\mathrm{TiO}_{2}$ and at $\mathrm{TiO}_{2}$ surfaces upon the irradiation of pump pulses at $620 \mathrm{~nm}$ whose photon energy is lower than that of excitation across the band gap. Most of the phonon modes observed are purely sine-like; the modes are excited by impulsive Raman scattering. However, a couple of phonon modes (at 200, 359, 440, and $823 \mathrm{~cm}^{-1}$ ) deviate from the sine-like oscillation. This implies that some real electronic transitions are involved in these coherent phonon generation. Since the excitation energy of pump pulses is below the band gap of $\mathrm{TiO}_{2}$, excitation across the band gap is not possible. Thus, they attributed these non-ISRS behavior to electronic transitions from an occupied midgap state derived from $\mathrm{Ti}^{3+}$ to the conduction band. It is interesting to note that contribution of the electronic excitation from the midgap state depends on the phonon mode. Some phonon modes do not show any influence of the electronic excitations from the midgap states, while the others do show the influence. This indicates that electron-phonon coupling surely depends on the phonon mode.

Next, we discuss how electron-phonon couplings are involved in the hole-trapping processes by focusing on bismuth vanadate $\left(\mathrm{BiVO}_{4}\right)$, a promising visible-light-driven photocatalyst. ${ }^{[59-63]} \mathrm{BiVO}_{4}$ is an inexpensive and robust semiconductor. $\mathrm{BiVO}_{4}$ has three crystal structures: scheelite structure with monoclinic and tetragonal systems, and zircon structure with a tetragonal system. ${ }^{[62]}$ Among these systems, a monoclinic scheelite phase of $\mathrm{BiVO}_{4}$ whose band gap is $2.4 \mathrm{eV}^{[60]}$ is active for oxygen evolution out of water oxidation under visible light irradiation. Although $\mathrm{BiVO}_{4}$ is not capable of reducing water, this can be combined with photocatalysts for hydrogen evolution to perform complete water splitting under visible light irradiation, i.e., the $\mathrm{Z}$ scheme. ${ }^{[64-67]}$ In this scheme, oxygen and hydrogen evolution reactions of each photocatalyst have to be coupled with electron mediators such as $\mathrm{Fe}^{2+} / \mathrm{Fe}^{3+}$ in aqueous solution. Substantial efforts have been made for improving the photocatalytic activity of $\mathrm{BiVO}_{4}$ using, for example, cocatalysts such as cobalt-phosphate. ${ }^{68,69]}$

$\mathrm{BiVO}_{4}$ powders and thin films of $\mathrm{BiVO}_{4}$ powders show a broad transient absorption (TA) band upon excitation at $400 \mathrm{~nm}$ in a wide probe wavelength range from 600 to 


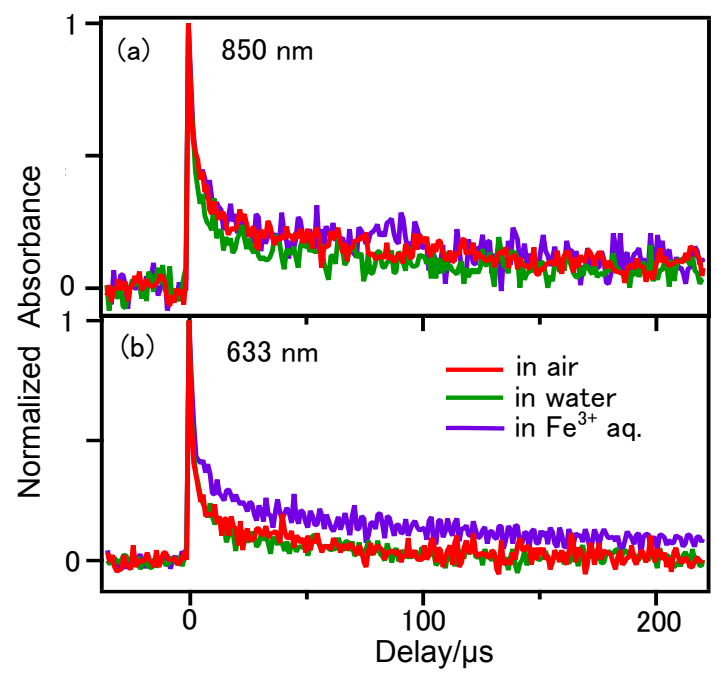

Figure 8: Time profiles of transient absorption of $\mathrm{BiVO}_{4}$ in delay times from 0 to $220 \mu \mathrm{s}$. Signal intensities are normalized at the peak values. Measurements were conducted for the sample in air, water, and $\mathrm{Fe}^{3+}$ aqueous solution. The pump wavelength is $400 \mathrm{~nm}$ and the probe wavelengths are (a) 850 and (b) $633 \mathrm{~nm}$. Reprinted with permission from Ref. ${ }^{[70]}$ Copyright 2013 American Chemical Society.

1300 nm. ${ }^{[70]}$ Because $\mathrm{BiVO}_{4}$ in the ground electronic state does not have any absorption bands at $\lambda>520 \mathrm{~nm},{ }^{[60]}$ the broad TA band originates in electrons and holes created by pump pulses. One of the drawbacks of transient absorption measurements of photocatalyst particles dispersed in water is that the origin of the transient signals is not obvious: it is not clear whether photo-generated-electrons or holes is responsible for the transient signals. To clarify the origin of TA signals, we compared the time profiles of transient absorption of $\mathrm{BiVO}_{4}$ in pure water with those in $\mathrm{Fe}^{3+}$ aqueous solution. $\mathrm{Fe}^{3+}$ is an electron scavenger; this is reduced to $\mathrm{Fe}^{2+}$ by photo-generated electron in $\mathrm{BiVO}_{4}$. With this method, we can determine the wavelength range contributed by electrons or holes; if the transient absorption in the wavelength range is due to photo-generated electrons, the decay of TA would be accelerated; if holes are responsible for the TA signals, the decay of TA would be decelerated, because the recombination rate of electron and hole becomes slower due to imbalance in the densities of charges created by the reduction of $\mathrm{Fe}^{3+}$. 
TA time profiles in the time range longer than sub- $\mu$ s clearly showed that the decay of TA was decelerated in the $\mathrm{Fe}^{3+}$ aqueous solution in the probe wavelength range from 600 to $800 \mathrm{~nm}$. In contrast, there were no differences in the decay profiles of TA at the wavelength longer than $850 \mathrm{~nm}$. Fig. 8 shows the TA time profiles measured at 633 and $850 \mathrm{~nm}$ in air, water, and an $\mathrm{Fe}^{3+}$ aqueous solution. At $633 \mathrm{~nm}$, the TA profile in the $\mathrm{Fe}^{3+}$ aqueous solution decays more slowly $\left(\tau_{1}=3.0 \pm 0.3 \mu \mathrm{s}\right.$ and $\left.\tau_{2}=150 \pm 8 \mu \mathrm{s}\right)$ than that in pure water, while the decay profiles with and without $\mathrm{Fe}^{3+}$ are identical within experimental error at $850 \mathrm{~nm}$. This indicates that photo-generated holes are responsible for the TA signals in the range from 600 to $800 \mathrm{~nm}$. In contrast, no influence of the electron scavengers on the time profiles in the longer wavelengths implies that carriers probed at the long wavelength is irrelevant to charge transfer at the photocatalyst surfaces. Thus, we attribute the absorption at $\lambda<800 \mathrm{~nm}$ to surface holes and that at $\lambda>800 \mathrm{~nm}$ to holes in the bulk. This assignment of the transient absorption band has been recently confirmed by Durrant and co-workers. ${ }^{[71]}$ They have measured transient absorption of a $\mathrm{BiVO}_{4}$ photoanode extending from 500 to $900 \mathrm{~nm}$ by using electron/hole scavengers and applied electric bias, concluding that the broad absorption band is attributed to not electrons but holes.

Holes responsible for the broad absorption band are likely trapped in the form of small polarons, whose energy levels are located at near the valence band maximum. Because the valence band mostly composed of $O 2 p$ states has a large width of $\sim 5 \mathrm{eV}$, ${ }^{[72]}$ the transitions from the occupied $O 2 p$ states to the hole states contribute to the broad absorption band. It is interesting to note that recent calculations of interpolaron optical transitions of anatase $\mathrm{TiO}_{2}$ have shown that the transition energy of holes in the surface layer is larger than that in the bulk, ${ }^{[73]}$ because the energy difference between the hole states involved in the interpolaron transition is larger at near the surface than in the bulk. Assuming the similar trend of energy of holes in $\mathrm{BiVO}_{4}$, we can understand why the TA at shorter wavelengths $(\lambda<800 \mathrm{~nm})$ is mainly contributed by surface holes, while that at longer wavelengths $(\lambda>800 \mathrm{~nm})$ is contributed by bulk holes.

Having established the wavelength range where surface trapped holes contribute, we 

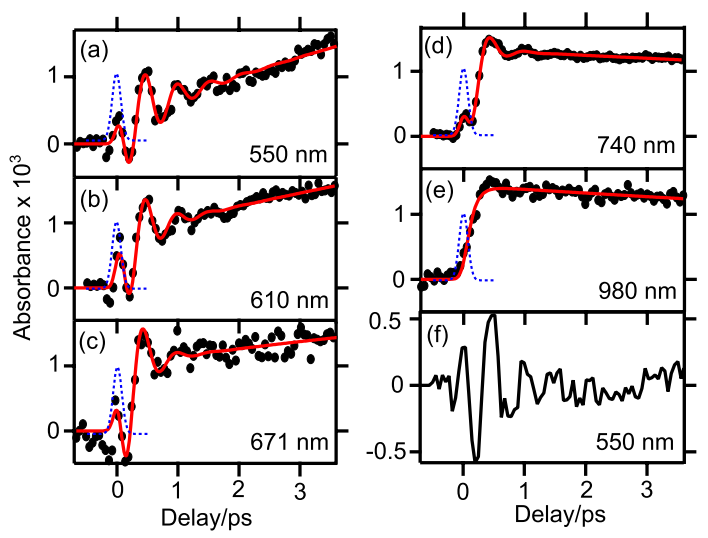

Figure 9: Time profiles of transient absorption of $\mathrm{BiVO}_{4}$ in the early delay time range up to 3 ps measured in air. The pump wavelength is $400 \mathrm{~nm}$ and the probe wavelengths are (a) 550, (b) 610, (c) 671, (d) 740, and (e) $980 \mathrm{~nm}$, and (f) the oscillating component deduced from the time profile at $550 \mathrm{~nm}$ by subtracting the rising background due to hole absorption. The blue dotted lines represent the time profile of pump pulse. Reprinted with permission from Ref. ${ }^{[70]}$ Copyright 2013 American Chemical Society.

examine transient absorption in the ps time range at $\lambda>550 \mathrm{~nm}$. Fig. 9 shows TA profiles within 3 ps. Although the TA profile at $980 \mathrm{~nm}$ has a rising component with $\tau_{1}=0.12$ ps, the TA time profiles at less than $700 \mathrm{~nm}$ clearly showed more complex rising edges. They contain slower rise components in addition to the one with $\tau_{1}=0.12$ ps: $\tau_{2}=12,19$, and $19 \mathrm{ps}$ at $\lambda=671,610$, and $550 \mathrm{~nm}$, respectively. Because the fastest rise component is compatible to the rising edge of the pump pulse, the almost instantaneous rise with $\tau_{1}=$ $0.12 \mathrm{ps}$ is attributable to holes in the valence band created through the transitions from the valence to the conduction band of $\mathrm{BiVO}_{4}$. In contrast, the slow rising components observed at $\lambda<700 \mathrm{~nm}$ likely originates in the filling process of hole trap states in the band gap.

Note that TA profiles at $\lambda<700 \mathrm{~nm}$ show the transient absorption signals exhibiting strong oscillating modulations superimposed on the slower rise components. These transient absorption profiles were convoluted with the pump laser pulse of a Gaussian time profile with a duration of $0.17 \mathrm{ps}$. The time constant of the faster rising component $\tau_{1}$ was 
fixed to $0.12 \mathrm{ps}$ and $\tau_{2}$ to $19 \mathrm{ps}(\lambda=550,610 \mathrm{~nm})$, or $12 \mathrm{ps}(\lambda=671 \mathrm{~nm})$. The fittings resulted in a frequency of oscillating component with $\sim 62 \mathrm{~cm}^{-1}(1.86 \mathrm{THz})$.

These oscillating signals are the manifestation of coherent phonons. What excitation is responsible for the generation of the coherent phonons? The filling process of hole trap sites with $\tau=10-20 \mathrm{ps}$ is too slow to generate the coherent phonons. Instead, these coherent phonons are generated by the electronic excitation across the band gap with pump pulses whose pulse duration is $0.17 \mathrm{ps}$; this is much shorter than the oscillation period of the phonon mode, 0.54 ps. Because the optical transitions across the band gap create electrons in the conduction band and holes in the valence bands, the equilibrium positions of ions are displaced in the excited states. Thus, a displacive force is exerted to ions in the bulk as well as at the surface, and hence ions start to move toward the new equilibrium positions right after the electronic transition.

It is interesting to note that the oscillating amplitude shows the rise and decay as shown in Fig. 8(f). Furthermore, the oscillating signals due to the coherent phonons only appear $\lambda<740 \mathrm{~nm}$. Because the background transient absorption in $\lambda<850 \mathrm{~nm}$ is proportional to the population of surface trapped holes, these facts indicate that the coherent phonon mode observed is strongly coupled with the electronic transitions associated with the surface trapped holes.

Avakyants et al. ${ }^{[7]}$ have reported the Raman spectra of single crystal $\mathrm{BiVO}_{4}$. They found 8 external modes: 62, 130, $212 \mathrm{~cm}^{-1}$ for $\mathrm{A}_{g}$ symmetry, 47, 55, 110, 144, $280 \mathrm{~cm}^{-1}$ for $\mathrm{B}_{g}$ symmetry; 8 internal modes: $326,370,386,711,830 \mathrm{~cm}^{-1}$ for $\mathrm{A}_{g}$ symmetry, 400, $642,743 \mathrm{~cm}^{-1}$ for $\mathrm{B}_{g}$ symmetry. Figure $10(\mathrm{a})$ is the comparison of Fourier-transformed power spectra of the oscillation component measured at 550 and $610 \mathrm{~nm}$ with the Raman spectrum of single crystal. The observed phonon band in this study has a frequency very close to the $\mathrm{A}_{g}$ mode of single crystal at $62 \mathrm{~cm}^{-1}$. As in Figure $10(\mathrm{~b})$, mainly $\mathrm{Bi}^{3+}$ oscillates with respect to $\mathrm{VO}_{4}^{3-}$ in this mode. The observed band is broader and the peak is shifted to higher frequency by $3 \mathrm{~cm}^{-1}$ from the band of single crystal. These differences could be due to inhomogeneity of hole trap sites. 
(a)

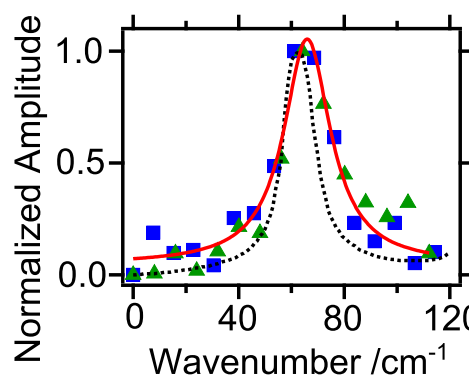

(b)

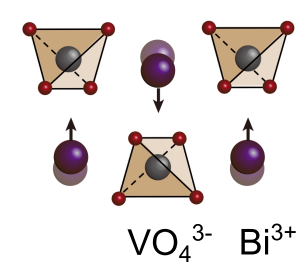

Figure 10: (a) Phonon spectra obtained by Fourier transformation of the oscillation component observed at 550 (blue) and 610 (green) nm. The dotted line is the Raman spectrum of $\mathrm{BiVO}_{4}$ reproduced from Ref. ${ }^{[74]}$ The solid line is the fitting result with the bulk Raman band and a Lorentzian line profile of a surface phonon band relevant to hole trap sites. (b) The schematic illustration of the $A_{g}$ external phonon mode at $62 \mathrm{~cm}^{-1}$. Reprinted with permission from Ref. ${ }^{[70]}$ Copyright 2013 American Chemical Society.

\section{Concluding remarks and outlook}

In this paper, we have discussed some illustrative examples of time-domain spectroscopy for observing ultrafast nuclear dynamics at surfaces of metal and metal oxides induced by electronic excitation. Upon electronic excitation with ultrashort laser pulses, nuclear motions are initiated through the electron-phonon couplings. These coherent nuclear motions can be monitored by observing both linear and nonlinear optical responses. Although the dynamics of coherent vibrational motions of molecules isolated in vacuum and in the condensed phase as well as coherent phonons in bulk materials have been extensively studied, coherent motions at the surfaces of solid materials are still relatively unexplored. This is because optical responses from the bulk usually overwhelm those from the surface. Thus, surface sensitive spectroscopies such as second-harmonic generation and sum frequency generation are suitable for this purpose. However, linear responses such as absorption and reflection can be used for probing surface dynamics if probe wavelengths are chosen such a way that the majority of optical responses at the wavelengths is limited to the surfaces.

There are two directions where the studies on electron-phonon couplings can be ex- 
tended: improvement in time and spatial resolutions. Obviously, as in the studies done for molecules in vacuum and in the condensed phase, applying pulses with much shorter widths, less than $10 \mathrm{fs}$, certainly extends the range of phonon mode frequencies much higher to be explored than the current studies. ${ }^{[5]}$ This provides much rich information of electron-phonon couplings that allow us to sort out vibration and phonon modes that can couple to electronic excitation of interest from those that do not couple to it. Second, improvement of spatial resolution is also important. Most of charge dynamics in photocatalysts have been studied by using either bulk single crystals or powders dispersed in liquid or thin films on substrates. Although measurements using powder photocatalysts are most realistic situations for heterogeneous photocatalysis, the optical responses are ensemble of powders with broad size distributions and with a wide variety of shapes with different facets. It is vital to know how charge separation/accumulation and hence redox reactions depend on the facet of crystallines for a clear understanding of the mechanism of heterogeneous photocatalysis. Studies combining optical probes with an optical microscope are now emerging ${ }^{[76,77]}$ and promise for providing more precise knowledge on photocatalysis. The ultimate goal for studies on electron-phonon couplings is to explore photoinduced processes of single adsorbate on solid surfaces with a fs time resolution. It needs more elaborations in methodology and advances in technique. In this context, an attempt at observing fs dynamics of a single molecule ${ }^{[78]}$ done recently is encouraging.

\section{Acknowledgments}

This work was supported by Grants-in-Aid for Scientific Research (S) (Grant No. 17105001) and (A) (Grant No. 25248006) from the Japan Society for the Promotion of Sciences. YM is grateful to co-workers particularly K. Watanabe, T. Yasuike, A. Kudo, and N. Aiga for the significant contributions of works cited in this paper.

\section{References}

[1] B. N. J. Persson and M. Persson. Solid State Commun., 1980, 36, 175-179. 
[2] M. Persson and B. Hellsing. Phys. Rev. Lett., 1982, 49, 662-665.

[3] K.-P. Bohnen, M. Kiwi, and H. Suhl. Phys. Rev. Lett., 1975, 34, 1512-1515.

[4] B. N. Persson and R. Ryberg. Phys. Rev. Lett., 1985, 54, 2119-2122.

[5] A. Okiji and H. Kasai. Surf. Sci. 1987, 188, L717-L722.

[6] M. Head-Gordon and J. T. Tully. Phys. Rev. B, 1992, 46, 1853-1856.

[7] J. C. Tully and M. Gomes. J. Vac. Sci. Technol. A, 1993, 11, 1914-1920.

[8] M. Brandbyge, P. Hedegård, T. F. Heinz, J. A. Misewich, and D. M. Newns. Phys. Rev. B 1995, 52, 6042-6056.

[9] B. N. J. Persson and R. Ryberg. Phys. Rev. Lett. 1982, 48, 549-552.

[10] B. N. J. Persson and R. Ryberg. Phys. Rev. B, 1985, 32, 3586-3596.

[11] B. N. J. Persson and E. Zaremba. Phys. Rev. B, 1985, 31, 1863-1872.

[12] H. Ueba. Prog. Surf. Sci., 1997, 55, 115-179.

[13] H. Ueba and M. Wolf. Science 2005, 310, 1774-1775.

[14] C. Frischkorn and M. Wolf. Chem. Rev., 2006, 106, 4207-4233.

[15] D. M. Riffe and A. J. Sabbah. Phys. Rev. B, 2007, 76, 085207-12.

[16] T. E. Stevens, J. Kuhl, and R. Merlin. Phys. Rev. B, 2002, 65, 144304-4.

[17] H. J. Zeiger, J. Vidal, T. K. Cheng, E. P. Ippen, G. Dresselhaus, and M. S. Dresselhaus. Phys. Rev. B, 1992, 45, 768-778.

[18] G. C. Cho, H. J. Bakker, T. Dekorsy, and H. Kurz. Phys. Rev. B, 1996, 53, 6904-6907.

[19] Y.-M. Chang, L. Xu, and H. W. K. Tom. Phys. Rev. Lett., 1997, 78, 4649-4652.

[20] K. Watanabe, D. T. Dimitrov, N. Takagi, and Y. Matsumoto. Phys. Rev. B 2002, 65, $235328-7$. 
[21] Y.-M. Chang, C. T. Chuang, C. T. Chia, K. T. Tsen, H. Lu, and W. J. Schaff. Appl. Phys. Lett., 2004, 85, 5224-5226.

[22] Y. Wang, L. Guo, X. Xu, J. Pierce, and R. Venkatasubramanian. Phys. Rev. B 2013, $\mathbf{8 8}, 064307-6$.

[23] H. P. Bonzel. Physics and Chemistry of Alkali Metal Adsorption. Elsevier, Amsterdam, 1989.

[24] E. Wimmer. J. Phys. F: Met. Phys., 1983, 13, 2313-2321.

[25] K. Watanabe, N. Takagi, and Y. Matsumoto. Chem. Phys. Lett., 2002, 366, 606-610.

[26] K. Watanabe, N. Takagi, and Y. Matsumoto. Phys. Rev. Lett., 2004, 92, 057401-4.

[27] K. Watanabe, N. Takagi, and Y. Matsumoto. Phys. Chem. Chem. Phys., 2005, 7, $2697-2700$.

[28] K. Watanabe, N. Takagi, and Y. Matsumoto. Phys. Rev. B, 2005, 71, 085414.

[29] Y. Matsumoto and K. Watanabe. Chem. Rev., 2006, 106, 4234-4260.

[30] M. Fuyuki, K. Watanabe, and Y. Matsumoto. Phys. Rev. B, 2006, 74, 195412-6.

[31] M. Fuyuki, K. Watanabe, D. Ino, H. Petek, and Y. Matsumoto. Phys. Rev. B, 2007, $\mathbf{7 6}, 115427-5$.

[32] K. Watanabe, K.-I. Inoue, I. F. Nakai, M. Fuyuki, and Y. Matsumoto. Phys. Rev. B 2009, 80, 075404-10.

[33] K. Watanabe, Y. Matsumoto, T. Yasuike, and K. Nobusada. J. Phys. Chem. A 2011, 115, 9528-9535.

[34] Y. R. Shen. The Principles of Nonlinear Optics. John Wiley, New Jersey, 2003.

[35] A. Liebsch. Electronic Excitations at Surfaces. Physics of Solids and Liquids. Plenum Press, 1997. 
[36] D. A. Arena, F. G. Curti, and R. A. Bartynski. Phys. Rev. B 1997, 56, 15404-15411.

[37] V. Chis, S. Caravati, G. Butti, M. I. Trioni, P. Cabrera-Sanfelix, A. Arnau, and B. Hellsing. Phys. Rev. B 2007, 76, 153404-4.

[38] S. I. Anisimov, B. L. Kapeliovich, and T. L. Perelman. Sov. Phys. JETP, 1974, 39, $375-377$.

[39] M. M. Wefers and K. A. Nelson. J. Opt. Soc. Am. B 1995, 12, 1343-1362.

[40] H. Katsuki, J. Delagnes, K. Hosaka, K. Ishioka, H. Chiba, E. Zijlstra, M. Garcia, H. Takahashi, K. Watanabe, M. Kitajima, Y. Matsumoto, K. Nakamura, and K. Ohmori. Nat Commun 2013, 4, 2801-7.

[41] A. Fujishima and K. Honda. Nature, 1972, 238, 37-38.

[42] M. R. Wasielewski. Acc. Chem. Res. 2009, 42, 1910-1921.

[43] K. Maeda and K. Domen. J. Phys. Chem. Lett. 2010, 1, 2655-2661.

[44] D. G. Nocera. Acc. Chem. Res. 2012, 45, 767-776.

[45] Y. Tachibana, L. Vayssieres, and J. R. Durrant. Nat. Photon. 2012, 6, 511-518.

[46] E. M. Bothschafter, A. Paarmann, E. S. Zijlstra, N. Karpowicz, M. E. Garcia, R. Kienberger, and R. Ernstorfer. Phys. Rev. Lett. 2013, 110, 067402-5.

[47] J. Chen, Y.-F. Li, P. Sit, and A. Selloni. J. Am. Chem. Soc. 2013, 135, 18774-18777.

[48] A. J. Cowan, J. Tang, W. Leng, J. R. Durrant, and D. R. Klug. J. Phys. Chem. C 2010, 114, 4208-4214.

[49] J. Tang, J. R. Durrant, and D. R. Klug. J. Am. Chem. Soc., 2008, 130, 13885-13891.

[50] M. A. Henderson. Surf. Sci. Rep., 2011, 66, 185 -297.

[51] G. M. Turner, M. C. Beard, and C. A. Schmuttenmaer. J. Phys. Chem. B 2002, 106, 11716-11719. 
[52] T. Yoshihara, R. Katoh, A. Furube, Y. Tamaki, M. Murai, K. Hara, S. Murata, H. Arakawa, and M. Tachiya. J. Phys. Chem. B, 2004, 108, 3817-3823.

[53] K. Iwata, T. Takaya, H.-O. Hamaguchi, A. Yamakata, T.-A. Ishibashi, H. Onishi, and H. Kuroda. J. Phys. Chem. B, 2004, 108, 20233-20239.

[54] A. Yamakata, T.-A. Ishibashi, and H. Onishi. Chem. Phys. 2007, 339, 133-137.

[55] Y. Tamaki, A. Furube, M. Murai, K. Hara, R. Katoh, and M. Tachiya. Phys. Chem. Chem. Phys., 2007, 9, 1453-1460.

[56] M. Xu, Y. Gao, E. M. Moreno, M. Kunst, M. Muhler, Y. Wang, H. Idriss, and C. Wöll. Phys. Rev. Lett. 2011, 106, 138302-4.

[57] T. Nomoto, A. Sasahara, and H. Onishi. J. Chem. Phys., 2009, 131, 084703-8.

[58] T. Nomoto and H. Onishi. J. Phys. Chem. C 2009, 113, 21467-21470.

[59] A. Kudo, K. Ueda, H. Kato, and I. Mikami. Catal. Lett., 1998, 53, 229-230.

[60] A. Kudo, K. Omori, and H. Kato. J. Am. Chem. Soc. 1999, 121, 11459-11467.

[61] S. Tokunaga, H. Kato, and A. Kudo. Chem. Mater. 2001, 13, 4624-4628.

[62] A. Iwase and A. Kudo. J. Mater. Chem., 2010, 20, 7536-7542.

[63] A. Iwase, H. Kato, and A. Kudo. J. Sol. Energy Eng., 2010, 132, 021106-5.

[64] H. Kato, M. Hori, R. Konta, Y. Shimodaira, and A. Kudo. Chem. Lett., 2004, 33, $1348-1349$.

[65] H. Kato, Y. Sasaki, A. Iwase, and A. Kudo. Bull. Chem. Soc. Jpn., 2007, 80, 24572464.

[66] A. Kudo and Y. Miseki. Chem. Soc. Rev., 2009, 38, 253-278.

[67] Y. Sasaki, H. Nemoto, K. Saito, and A. Kudo. J. Phys. Chem. C 2009, 113, 1753617542 . 
[68] D. Wang, R. Li, J. Zhu, J. Shi, J. Han, X. Zong, and C. Li. J. Phys. Chem. C 2012, 116, 5082-5089.

[69] F. F. Abdi and R. V. d. Krol. J. Phys. Chem. C 2012, 116, 9398-9404.

[70] N. Aiga, Q. Jia, K. Watanabe, A. Kudo, T. Sugimoto, and Y. Matsumoto. J. Phys. Chem. C 2013, 117, 9881-9886.

[71] Y. Ma, S. R. Pendlebury, A. Reynal, F. Le Formal, and J. R. Durrant. Chem. Sci., 2014 .

[72] L. Zhang, D. Chen, and X. Jiao. J. Phys. Chem. B 2006, 110, 2668-2673.

[73] P. Zawadzki. J. Phys. Chem. C 2013, 117, 8647-8651.

[74] L. P. Avakyants, A. V. Chervyakov, V. S. Gorelik, and P. P. Sverbil. Journal of Russian Laser Research 2004, 25, 535-580.

[75] T. Kobayashi, T. Saito, and H. Ohtani. Nature, 2001, 414, 531-534.

[76] T. Tachikawa, S. Yamashita, and T. Majima. J. Am. Chem. Soc. 2011, 133, 71977204.

[77] T. Tachikawa, T. Yonezawa, and T. Majima. ACS Nano, 2013, 7, 263-275.

[78] J. Lee, S. M. Perdue, A. Rodriguez Perez, and V. A. Apkarian. ACS Nano 2014, 8, $54-63$. 